\section{Neonatal Thyrotoxicosis Co-existing With Early Onset Sepsis}

Neonatal thyrotoxicosis is a rare disorder, and still rarer is its cooccurrence with early onset neonatal sepsis. Thyroid dysfunction, both hypothyroidism and hyperthyroidism, can alter a range of immune functions including chemotaxis, phagocytosis, production of reactive oxygen species, and cytokine production [1].

A 31-week-old appropriate for gestational age, baby girl weighing $1420 \mathrm{~g}$ was born to gravida 2 mother by precipitate labor. Mother was an unbooked case and presented to our hospital in labor with meconium stained amniotic fluid. Apgar scores were 7 and 8 at 1 and 5 minute, respectively. On admission baby had severe respiratory distress with Silverman Anderson score of 8 . She was noted to have tachycardia with a heart rate of 198/per minute. Due to non-availability of ventilator, she was manually ventilated for 6 hours. We kept the differential diagnosis of early onset sepsis, respiratory distress syndrome and meconium aspiration syndrome. She received injectable ampicillin and gentamicin and provided supportive care. On investigation, her total leucocyte count was $3675 \times 10^{9}$ / $\mathrm{L}$, platelets $8900 \times 10^{9} / \mathrm{L}$ and procalcitonin levels $7.28 \mu \mathrm{g} / \mathrm{L}$ (normal, less than $0.5 \mu \mathrm{g} / \mathrm{L}$ ). Chest $X$-ray showed cardiomegaly and bilateral clear lung fields. Blood culture grew Enterococcus faecalis sensitive to ampicillin and gentamicin.

After 6 hours of age baby showed improvement and was shifted to continuous positive airway pressure (CPAP). However, she was noted to have persistent tachycardia, with heart rates ranging from 180-210 per minute without any evidence of poor tissue perfusion. Electrocardiogram showed sinus tachycardia. Echocardiography demonstrated structurally normal heart. Detailed examination of baby revealed a swelling over anterior aspect of neck. The eyes of the baby were prominent. Baby appeared irritable and hyperalert. Mother gave history of neck swelling for 3 months and tremors prior to delivery. She had exophthalmos and tachycardia. Thyroid profile of baby on day 3 of life in venous blood showed triiodothyronine (T3) levels of $315.4 \mathrm{ng} / \mathrm{dL}$, thyroxine (T4) levels of $19.5 \mu \mathrm{g} / \mathrm{dL}$ and thyroid stimulating hormone (TSH) levels of $<0.01 \mathrm{mU} / \mathrm{mL}$. Mother's thyroid profile showed a free T3 value of $10.2 \mathrm{pmol} / \mathrm{L}$, free T4 value of $80.4 \mathrm{pmol} / \mathrm{L}$ and TSH value of $<0.01 \mathrm{mU} / \mathrm{mL}$. Ultrasound of neck of the baby showed bulky thyroid gland with altered echotexture, suggestive of thyroiditis. TSH receptor antibody levels in baby were 37.75 IU/L (normal, less than 1.75 IU/L). The final diagnosis was early onset sepsis with neonatal thyrotoxicosis.

We started oral propranolol $2 \mathrm{mg} / \mathrm{kg} /$ day in three divided doses along with methimazole $0.5 \mathrm{mg} / \mathrm{kg} /$ day in three divided doses from day 3. Tachycardia settled after 72 hours of treatment following which propranolol was tapered. Thyroid function tests were repeated after 7 days of starting methimazole which showed no significant improvement, hence dose of methimazole was increased. On follow-up at 6 weeks and 3 months of age, baby showed normal growth and development. Thyroid functions normalized by 18 weeks of age. Methimazole dose was tapered and stopped. Baby tolerated methimazole well and did not show any adverse drug reaction.

In the present case, neonatal thyrotoxicosis coexisted with early onset sepsis which made diagnosis challenging. Although, free $\mathrm{T} 4$ could not be done in the postnatal period in the index case, rest of the investigations and clinical examination findings of the mother and baby were suggestive of neonatal thyrotoxicosis. Thyroid hormones are known to act as modulators of immune response [1] and it is possible that thyrotoxicosis predisposed the baby to early onset sepsis. The clue to diagnosis of thyrotoxicosis was persistent tachycardia in the absence of any known cause, such as tachyarrhythmia or shock.

Previous studies on pregnant women with thyrotoxicosis showed that women treated earlier in pregnancy were more likely to be euthyroid at delivery and had better pregnancy outcomes [2]. Uncontrolled thyrotoxicosis in pregnancy is associated with abortion, prematurity, low birthweight, and stillbirth [2]. Polak, et al. [3] reported clinical manifestations on 10 neonates with congenital thyrotoxicosis. Nine of them were due to autoimmune causes. Tachycardia was the commonest sign followed by goiter and hyperexcitability. Our patient responded well to treatment. Tachycardia settled first, followed by hyperexcitability. Neck swelling gradually reduced in size on follow-up but eye signs persisted longer. Thyroid function returned to normal by 4.5 months of age. The present case shows the rare occurrence of neonatal sepsis with congenital thyrotoxicosis, and highlights the importance of maternal history and examination in making correct neonatal diagnosis.

RimJHim SONOWAL, *AKANKSHa ANJALI AND ASHOK KUMAR From Division of Neonatology, Department of Pediatrics, Institute of Medical Sciences, Banaras Hindu University, Varanasi, India. *rimjhimsonowal@yahoo.in.

\section{REFERENCES}

1. Vito PD, Incerpi S, Pedersen JZ, Luly P, Davis FB, Davis PJ. Thyroid hormones as modulators of immune activities at the cellular level. Thyroid. 2011;21:879-90.

2. Davis LE, Lucas MJ, Hankins GDV, et al. Thyrotoxicosis complicating pregnancy. Am J Obstet Gynecol. 1989;160:63-70.

3. Polak M, Legac I, Vuillard E, Guibourdenche J, Castanet M, Luton D. Congenital hyperthyroidism: The fetus as a patient. Horm Res. 2006;65:235-42. 